\title{
Variante C.113 C>T (P.SER 38PHE) de significado incierto asociada con caso típico de MEN1
}

\author{
Variant C.113 C>T (P.SER 38PHE) of uncertain significance associated \\ with typical MEN1
}

\author{
Vargas $Y^{1}$.
}

${ }^{1}$ Médica Endocrinóloga, Universidad Nacional.

Autor de correspondencia: Yolanda Vargas Pérez

Correo electrónico: kadi17co@gmail.com

Fecha de recepción: 20/06/2018

Fecha de aceptación: 10/04/2019

\section{Resumen}

La neoplasia endocrina múltiple es una condición hereditaria, con herencia autosómica dominante, que tiene alta penetrancia y está relacionada con mutaciones del gen supresor de tumores MEN1. Se caracteriza por la presencia de tumores en las glándulas endocrinas, aunque también puede estar asociada con otros tumores, como lipomas, angiofibromas y colagenomas. Este gen tiene múltiples variantes descritas y no hay una clara relación genotipo fenotipo. Hay algunas variantes descritas como de significado incierto. Se reporta un caso de MEN1 típico asociado con una variante descrita como de significado incierto, la misma variante fue encontrada en uno de los hijos de la paciente. La importancia del reporte está en que al aumentar los casos descritos asociados con una misma variante se podría determinar su patogenicidad y podría servir también como guía para realizar asesoría genética.

Palabras clave: neoplasia endocrina múltiple tipo 1 , tumores de la glándula paratiroides, tumores pancreáticos, tumores neuroendocrinos, lipomas, variante genética.

\section{Abstract}

Multiple endocrine neoplasia is an inherited predisposition to parathyroid, endocrine pancreas, pituitary, adrenal and neuroendocrine tumors. It segregates as an autosomal dominant disease with high penetrance and is related to mutations in the MEN1 gene. This condition has clinical variability as many glands and organs can be involved, and is important to clarify the relation between specific mutations and specific phenotypes. There are some pathogenic variants of the gen that have been described. Some are considered of uncertain significance and others have the category of probably pathogenic. There is not absolutely correlation between gene variants and phenotype. This is a case of MEN 1 with the variant c.113 C>T (p.Ser 38Phe), reported as of uncertain significance. The patient present with phenotype of MEN1 and multiple pancreatic lipomas. The same mutation was identified in one of her sons.

Keywords: Multiple Endocrine Neoplasia Type 1, parathyroid neoplasms, pancreatic neoplasms, neuroendocrine tumors, lipomas, genetic variant.

\section{Introducción}

La neoplasia endocrina múltiple es una entidad clínica que se caracteriza por la predisposición hereditaria a tumores de paratiroides, páncreas endocrino, hipófisis, suprarrenales y tejido neuroendocrino. También se describen tumores de tejidos blandos como lipomas, angiofibromas y colagenomas. Se segrega como una enfermedad autosómica dominante con alta penetrancia y está relacionada con mutaciones en el gen MEN1. Esta afección tiene variabilidad clínica, ya que muchas glándulas y órganos pueden estar involucrados y es importante aclarar la relación entre variantes genéticas específicas y fenotipos específicos ${ }^{(1)}$. Existen algunas variantes patogénicas del gen que se han descrito. Algunos se consideran de significado incierto y otros tienen la categoría de probablemente patogénicos. No hay una correlación absoluta entre las variantes genéticas y el fenotipo. Este es un caso de MEN1 con la variante c.113 C> T (p.Ser 38Phe), reportada como de significado incierto $^{(2)}$. La paciente presenta fenotipo de MEN1 y múltiples lipomas pancreáticos. La misma mutación se identificó en uno de sus hijos. 


\section{Presentación del caso}

Mujer de 38 años de edad que consulta por primera vez en julio de 2015 debido a aumento progresivo de peso, hinchazón, edema palpebral, dolor de cabeza crónico diario y síntomas de fatiga crónica. Como antecedentes clínicos presentó una fractura de metatarso por traumatismo de bajo impacto. Su historia familiar no tiene datos relevantes para tumores o tumores neuroendocrinos familiares. Tenía ciclos regulares con sangrado abundante y diagnóstico previo de ovarios poliquísticos. La paciente tiene dos hijos sanos. Se realizaron estudios iniciales y se encontraron niveles altos de calcio $(11,5$ $\mathrm{mg} / \mathrm{dL}$ ) asociados con un aumento de iPTH $(176,6 \mathrm{pg} / \mathrm{mL}) \mathrm{y}$ bajo nivel de fósforo $(1,9 \mathrm{mg} / \mathrm{dL})$.

Se realizó una gammagrafía de paratiroides, la cual reveló un adenoma paratiroideo y la paciente fue llevada a cirugía. El informe de patología fue adenoma paratiroideo. Luego de la cirugía persistió con hipercalcemia y una iPTH elevada. Se realizó nueva gammagrafía paratiroidea y por hallazgo positivo fue llevada nuevamente a cirugía. El informe de patología esta vez fue hiperplasia paratiroidea. De forma simultánea, fue vista por ginecología por sangrado genital abundante durante la menstruación, deficiencia de hierro y antecedente de ovarios poliquísticos. Un ultrasonido vaginal reveló líquido libre en la cavidad peritoneal, por lo cual se decidió tomar una tomografía computarizada de abdomen; esta reveló una imagen multiquística focal en la región de la cola del páncreas de $21 x$ 25 x $26 \mathrm{~mm}$, cálculos renales intrarrenales de menos de $4 \mathrm{~mm}$ y líquido peritoneal libre.

Se tomó una resonancia magnética (RM) abdominal que reveló dos lesiones focales en la cola del páncreas: una microquística con tabique, sin componente sólido de $2 \times 2 \mathrm{~cm}$, y otra de $1 \mathrm{~cm}$; adicional de una imagen suprarrenal sugestiva de adenoma de menos de $1 \mathrm{~cm}$. La lesión en la cola del páncreas se consideró un tumor neuroendocrino. De acuerdo con el tamaño del tumor inicialmente se consideró abordaje no quirúrgico, sin embargo, se realizó una ecografía endoscópica y una biopsia pancreática guiada. La imagen mostró una lesión mixta quística sólida multiseptada de 26,2 x 14,7 x 41,9 mm y otra en la cola del páncreas de 4,5 mm.

El informe de la biopsia fue un tumor neuroendocrino de bajo grado basado en Ki67. Se tomaron pruebas de laboratorio complementarias con prolactina, IGF 1, cortisol urinario libre, gastrina, ácido hidroxindolacético y cromogranina A. La prolactina mostró una elevación leve (30,13 ng/mL). Los demás laboratorios no mostraron datos relevantes. Una resonancia magnética de la hipófisis reveló hiperintensidad, que se interpretó como pico adenoma de $3 \mathrm{~mm}$. Debido a los resultados de la ecografía endoscópica, la paciente fue llevada a pancreatectomía distal y esplenectomía. El informe de patología fue un tumor localizado en el cuerpo y la cola del páncreas, multifocal, patrón trabecular acinar bien diferenciado y mixto quístico-sólido. No mitosis. Extensión tumoral microscópica: (T2) tumores múltiples mayores de $2 \mathrm{~cm}$ limitados al páncreas. Márgenes de resección negativos. Ausencia de invasión perineural, linfática y vascular. Un ganglio linfático negativo. Tamaño de los tumores $4 ; 1,4 ; 0,4$ y 0,4 cm. Múltiples lipomas, el más grande de más de $2 \mathrm{~cm}$. Hiperplasia de células neuroendocrinas ductales. Sinaptofisina y cromogranina (+) en células neuroendocrinas neoplásicas. CD56: débilmente positivo en células neoplásicas de origen neuroendocrino. Ki67: 1\%. Bajo grado bien diferenciado NET.

Se realizó estudio genético de gen MEN1 y reveló una variante de significación incierta de c.113 C>T (p.Ser38Phe), probablemente patogénica. Se realizó tamizaje con calcio sérico a sus hijos y uno de ellos mostró nivel de calcio normal alto. Se realizó estudio genético a sus hijos y aquel con el calcio normal alto mostró la misma variante del gen.

\section{Discusión}

La neoplasia endocrina múltiple tipo 1 es una enfermedad genética asociada con mutaciones del gen MEN1. Esta tiene alta penetrancia y más del $95 \%$ de los pacientes tendrá síntomas a la edad de 40 años. Hay gran variabilidad en la edad de presentación, los tipos de tumores y también en el curso clínico y severidad de la enfermedad. La heterogeneidad de las variantes y mutaciones en el gen MEN1 hace difícil establecer una correlación precisa entre el genotipo y el fenotipo ${ }^{(1)}$.

El diagnóstico clínico de la neoplasia endocrina múltiple se realiza por el hallazgo de dos tumores endocrinos, por lo general, en paratiroides, hipófisis anterior y gastroenteropancreáticos. El análisis genético detecta variantes patogénicas heterocigotas del gen MEN1 hasta en el 80\%-90\% de los casos en síndrome familiar y hasta en $65 \%$ en casos aislados ${ }^{(1)}$.

El gen MEN1 es un gen supresor tumoral que codifica para una proteína nuclear llamada menina y tiene gran cantidad de interacciones con proteínas relacionadas con la regulación de la transcripción, estabilidad genómica, proliferación y división celular. Se cree que las mutaciones en el gen MEN1 alteran estas interacciones y afectan puntos críticos de la división y proliferación celular. Las mutaciones del gen son inactivadoras y hasta el 90\% de los tumores tiene pérdida de la heterocigosidad. Se han encontrado más de 1300 variantes del gen sin lograrse hasta la fecha establecer correlación fenotipo genotipo ${ }^{(2-6)}$.

El análisis de la secuencia del gen MEN1 detecta variantes que pueden ser benignas, probablemente benignas, de significado incierto, probablemente patogénicas o patogénicas ${ }^{(1,3)}$. Esta clasificación se encuentra estandarizada y se realiza con base en los reportes enviados de diferentes laboratorios en el mundo y se puede consultar en la base de datos ClinVar. En este caso, el hallazgo fue el de una variante de significado incierto, 
lo cual significa que puede o no ser causante de la enfermedad y puede o no estar asociada con un riesgo aumentado de enfermedad o con un fenotipo anormal ${ }^{(3)}$. El hallazgo de estas variantes no confirma ni descarta el diagnóstico ${ }^{(1,3)}$. Sin embargo, es importante reportar estas variantes porque de ello depende el cambio de clasificación en el tiempo. Con el resultado del análisis genético, en este caso se realizó la búsqueda en ClinVar y se encontró que si bien la variante esta reportada, solo existe un único reporte y no tiene datos clínicos anexos.

Los tumores de paratiroides son la principal endocrinopatía asociada con MEN1. La mayoría de pacientes se presenta con hipercalcemia antes de los 50 años. Aunque esta paciente no consultó específicamente por síntomas asociados con hipercalcemia, tenía una historia previa importante de fractura con trauma mínimo y esto generó el estudio del metabolismo del calcio y, a partir de allí, los otros hallazgos descritos. Los tumores pancreáticos pueden estar entre el 30\%-80\% de los pacientes con MEN1 y usualmente son la siguiente manifestación después del hiperparatiroidismo ${ }^{(2,4)}$. Pueden ser funcionantes o no. En este caso, los tumores fueron detectados con imágenes y no se definió su funcionalidad. Si bien la paciente no tenía síntomas claros de hiperfunción hormonal es posible que la detección temprana haya evitado la progresión a manifestaciones clínicas. No se realizó inmunohistoquímica de los tumores pancreáticos.

Los más relevantes clínicamente son los tumores endocrinos, tanto por la funcionalidad como por la posibilidad de malignidad. Sin embargo, la presencia de otros tumores, como los lipomas asociados, se reporta hasta en el 30\% de los casos, siendo su localización más frecuente subcutánea y más rara la localización visceral, como en este reporte de caso $^{(5)}$. Se han realizado estudios genéticos en lipomas de pacientes afectados por MEN1 y en pacientes con lipomas esporádicos y en los dos se han encontrado alteraciones del gen MEN1, aunque no en todos los casos. Esto sugiere que, aunque las mutaciones sean compartidas, las vías de tumorogénesis pueden ser diferentes para los tumores endocrinos ${ }^{(5)}$. Sin embargo, los estudios son pequeños y no hay relación clara del genotipo con la presencia o no de lipomas. En este caso llama la atención la presencia de múltiples lipomas en páncreas en ausencia de lipomas subcutáneos.

Identificar la variante genética específica tiene, a largo plazo, el beneficio de poder establecer una relación genotipofenotipo. Si aumentan los reportes de esta variante asociada específicamente con lipomas se podrá determinar esta correlación. Asimismo, establecer la variante genética en la paciente hizo que se realizara el estudio genético en los dos hijos y uno de ellos tiene la misma variante. Esto es parte de lo recomendado en asesoría genética ${ }^{(6)}$. En este momento, los hermanos de la paciente y sus padres se encuentran en seguimiento clínico para establecer si hay otros casos de tumores endocrinos familiares no diagnosticados.

\section{Conclusiones}

La neoplasia endocrina múltiple tipo 1 tiene un amplio espectro de manifestaciones clínicas. Cuando los síntomas no son tan evidentes, como en este caso, la sospecha clínica y los antecedentes pueden orientar el diagnóstico.

Esta es una enfermedad causada por mutaciones del gen MEN1, del cual se conocen múltiples variantes, de las que solo algunas tienen reportes de patogenicidad y otras, como en este caso, apenas están descritas. Se desconoce hasta ahora la correlación genotipo fenotipo.

El diagnóstico en un miembro de la familia orienta a buscar en otros la presencia de manifestaciones patológicas para ofrecer asesoría genética, incluso ante la ausencia de síntomas. En este caso, en uno de los hijos de la paciente se encontró la misma variante y presentó calcio elevado al tamizaje.

Al existir cada vez más reportes es posible que en el futuro esta variante pueda considerarse patogénica y estos datos pueden ser útiles para el asesoramiento genético familiar y el tratamiento temprano.

\section{Conflictos de interés}

Ninguno.

\section{Referencias}

1. Landrum MJ, Lee JM, Benson M, Brown G, Chao C, Chitipiralla S, et al. ClinVar: public archive of interpretations of clinically relevant variants. $\mathrm{Nu}$ cleic Acids Res. 2016;44(D1):D862-8.

2. Syro LV, Scheithauer BW, Kovacs K, Toledo RA, Londoño FJ, Ortiz LD, et al. Pituitary tumors in patients with MEN1 syndrome. Clinics, 2012;67(S1):43-8.

3. Giusti F, Marini F, Brandi ML. Multiple endocrine neoplasia type 1. En: Adam MP, Ardinger HH, Pagon RA, et al. (editores). GeneReviews [Internet]. Seattle (WA): University of Washington. 1993-2018.
4. Li J, Zeng L, Yang Y, Zhan Y, Tao J, Wu B. Multiple endocrine neoplasia type 1 - presenting multiple lipomas and hypoglycemia onset. Am J Case Rep, 2012:13:224-9.

5. Agarwal S, Monsaert R. Multiple endocrine neoplasia type I and lipomas. Hosp Physic. 2002;38:51-4.

6. Taboada LB, Vera A, Kattah W, López R, Medina VL, González D. Mutación del gen de la menina: desde el hiperparatiroidismo familiar aislado a la neoplasia endocrina múltiple de tipo 1. Rev Colomb Cir. 2011;26:118-30. 